\section{Robust Estimation and Control of Tire Traction Forces}

Tesheng Hsiao, Member, IEEE

\begin{abstract}
Due to increasingly demanding requirements for driving safety, precise and robust control of tire traction forces is essential to implement high-performance vehicle-control systems. However, traditional slip-ratio-based methods cannot guarantee achievement of desired traction forces due to a lack of tire force feedback. This paper proposes an observer-based traction force control scheme that is robust with respect to variations of road conditions and tire model uncertainties. Moreover, achievement of desired traction forces is guaranteed. Simulation results verify the robust and satisfactory performance, even under suddenly changing road conditions, as well as parametric and nonparametric tire model uncertainties.
\end{abstract}

Index Terms-Tire force control, tire force estimation, traction control.

\section{INTRODUCTION}

Driving safety and performance can be significantly improved if tire traction forces are under careful control. However, control of tire traction forces is challenging in many aspects. First, the tire-road interaction is complicated, highly nonlinear, and uncertain. Many tire models have been proposed [1], but each of them considers only parts of tire behavior (e.g., speed dependence). Moreover, it is difficult to know in advance or to estimate online the parameters associated with the tire models. Therefore, tire-model-based control schemes inevitably suffer from considerable uncertainties from both model parameters and model structures. Robust control methods such as sliding-mode control and/or fuzzy logic control were investigated in [2]-[4]. Variants of sliding-mode control such as the second-order sliding-mode control [5], [6] and the dynamic surface control [7] were also explored for their good robustness and reduced chattering phenomenon.

The second challenge is that the operational conditions of tires are time varying and may substantially change. In particular, traction forces significantly depend on road conditions. To deal with this problem, some traction force control systems estimated the tire-road friction coefficient online using the recursive least square algorithm [2] or the sliding-mode observer [5], [8].

The third challenge comes from immeasurability of tire traction forces; thus, traction force control is indirectly accomplished by slip ratio control. Consequently, the control objectives of slip-ratio-based methods focus on tracking the desired slip ratio [2], [6], attaining maximum traction forces [2], [3], [5], [8], or stabilizing the slip ratio under all road conditions [7].

However, the ever-demanding requirements for driving safety inspire the need for precise traction force control. Recent studies have shown that coordinating traction forces of all tires in an optimal way improves yaw stability of the vehicle in severe situations [9]-[12]. Unfortunately, perfect slip ratio control does not guarantee achievement of desired traction forces because nonparametric tire model

Manuscript received November 17, 2011; revised March 29, 2012; July 24, 2012; and October 4, 2012; accepted October 30, 2012. Date of publication November 30, 2012; date of current version March 13, 2013. The review of this paper was coordinated by Dr. S. Anwar.

The author is with the Department of Electrical and Computer Engineering, National Chiao Tung University, Hsinchu 30010, Taiwan (e-mail: tshsiao@cn.nctu.edu.tw).

Color versions of one or more of the figures in this paper are available online at http://ieeexplore.iee.org.

Digital Object Identifier 10.1109/TVT.2012.2230656 uncertainties hamper correct transfer of the desired traction force to the target slip ratio. To solve the problem, tire force estimators were investigated [13]-[16]. However, observer-based traction force control needs further exploration for guaranteed robust stability and performance. On the other hand, working prototypes of tire force sensors were utilized for firing different phases of the slip ratio control law [17], instead of directly controlling the traction force.

This paper proposes an observer-based traction force control scheme that guarantees achievement of the desired traction force and is robust with respect to tire model uncertainties, as well as variations in road conditions. The proposed controller accepts the traction force command from an "upper-level" vehicle-control system [9]-[12] and delivers the required wheel torque to attain the desired traction force. In addition, maximum traction force is estimated online to prevent the controller from tracking an unachievable force command.

The robust performance of the proposed controller was verified by simulations and compared with those of an "ideal" slip-ratiobased controller and a simple traction force estimator in [15]. The results show that the proposed method achieves superior performance, regardless of sudden changes in road conditions, as well as parametric and nonparametric tire model uncertainties.

The rest of this paper is organized as follows: Section II introduces the tire models used in this paper. Section III presents the robust traction force estimator. The control algorithm is proposed in Section IV. Simulation results are presented in Section V, and Section VI concludes this paper.

\section{TIRE MODELS}

In this paper, we assume that the vehicle is traveling along a straight line without cornering. Neglecting the rolling resistance and deformation of the tire, we have $I_{w} \dot{\omega}=T-F_{x} R$, where $T$ is the wheel torque, which is the sum of the braking torque and the driving torque from the engine. $T$ is positive for acceleration and negative for braking. $I_{w}, R, \omega$, and $F_{x}$ are the moment of inertia, radius, rotational velocity, and traction force of the wheel, respectively. $\omega$ is positive when the vehicle is moving forward, and $F_{x}$ is positive during acceleration.

The traction force $F_{x}$ is closely related to the tire's slip ratio, which is defined as $\lambda=\left(R \omega-v_{w}\right) / \max \left\{R \omega, v_{w}\right\}$, where $v_{w}$ is the translational velocity of the wheel. One of the most popular tire models characterizing the relation between $F_{x}$ and $\lambda$ is the magic formula [18]

$$
\begin{aligned}
F_{x}=f_{M}\left(\lambda, \mu_{p}, F_{z}\right) & \\
=\mu_{p} D_{M} \sin \left\{C_{M} \tan ^{-1}[\right. & B_{M} \lambda-E_{M} \\
& \left.\left.\times\left(B_{M} \lambda-\tan ^{-1}\left(B_{M} \lambda\right)\right)\right]\right\}
\end{aligned}
$$

where $\mu_{p}$ is the tire-road friction coefficient, and $F_{z}$ is the normal tire force. $F_{z}$ is not a constant due to the weight transfer effect. $B_{M}, C_{M}$, $D_{M}$, and $E_{M}$ are parameters associated with the magic formula whose values vary with $F_{z}$. Another widely used tire model is the Dugoff model [19]

$$
F_{x}=f_{D}\left(\lambda, \mu_{p}, F_{z}\right)=C_{x} \frac{\lambda}{1-|\lambda|} k(\sigma)
$$

where $\sigma=\left(\mu_{p} F_{z}(1-|\lambda|)\right) /\left(2 C_{x}|\lambda|\right), \quad k(\sigma)=1$ if $\sigma \geq 1$, and $k(\sigma)=\sigma(2-\sigma)$ if $\sigma<1$. $C_{x}$ is the longitudinal stiffness of the tire. 
The $F_{x}-\lambda$ relations based on (1) and (2) for various $\mu_{p}$ and $F_{z}$ are shown in Fig. 1, and we can observe that (1) and (2) are similar for small slip ratios but significantly different near the limitation of the traction force.

In a general slip-ratio-based control system, the desired traction force $F_{x d}$ is converted to the target slip ratio $\lambda_{d}$ through an inverse nominal tire model, and the controller tracks the target slip ratio. Although the slip ratio tracking is perfect, i.e., $\lambda=\lambda_{d}$, the desired traction force may not be attained due to tire model uncertainties. Parametric model uncertainties can be compensated for by online estimation of the tire-road friction coefficient [2], [5] and tire model parameters [20], [21]; however, compensation for the nonparametric model uncertainties remains unsolved. To emphasize the robustness of the proposed control scheme with respect to both parametric and nonparametric tire model uncertainties, we choose the Dugoff model with fixed $C_{x}, \mu_{p}$, and $F_{z}$ as the nominal tire model for controller design and assume that the actual tire model is unknown. In the simulation, we use the magic formula as the actual tire model.

\section{TRACTION ForCE Estimation}

\section{A. Design of the Estimator}

We assume that $F_{x}=f\left(\lambda, \mu_{p}, F_{z}\right)$, where $f$ is an unknown function satisfying $\partial f\left(\lambda, \mu_{p}, F_{z}\right) / \partial \lambda=(1+\Delta)\left(\partial f_{0}(\lambda) / \partial \lambda\right)$, and $|\Delta| \leq \bar{\Delta}$ for all $\lambda, \mu_{p}$, and $F_{z}$, where $f_{0}(\lambda)=f_{D}\left(\lambda, \mu_{p 0}, F_{z 0}\right)$ is the Dugoff model evaluated at fixed constants $\mu_{p 0}$ and $F_{z 0} . \Delta$ is the model uncertainty and is assumed to be bounded by a known constant $\bar{\Delta}$. To avoid the potential numerical problem introduced by the large slope $\partial F_{x} / \partial \lambda$ for small $\lambda$, we use the normalized traction force $\mu=F_{x} / F_{z 0}$ in the subsequent derivation. $\mu_{p}$ is regarded as quasistatic; hence, we have $\dot{\mu}=\left(1 / F_{z 0}\right)\left[(\partial f / \partial \lambda) \dot{\lambda}+\left(\partial f / \partial F_{z}\right) \dot{F}_{z}\right] \triangleq$ $(1+\Delta) B\left(\mu, \lambda, v_{w}, \omega, T\right)+\left(\partial f / \partial F_{z}\right)\left(\dot{F}_{z} / F_{z 0}\right)$, where $B\left(\mu, \lambda, v_{w}\right.$, $\omega, T)=\left(\partial f_{0} / \partial \lambda\right)\left((1-|\lambda|) / F_{z 0}\right)\left[\left(\left(T-\mu F_{z 0} R\right) / \omega I_{w}\right)-\left(\dot{v}_{w} / v_{w}\right)\right]$.

Assume that $\omega, v_{w}, \dot{v}_{w}$, and $T$ are available, then the proposed traction force estimator is

$$
\begin{aligned}
\dot{\hat{\mu}} & =\hat{B}+\eta\left(\phi, \hat{\omega}, \hat{\omega}_{f}\right) \\
\dot{\phi} & =\Phi \\
\dot{\hat{\omega}} & =\frac{T-\hat{\mu} F_{z 0} R}{I_{w}}+a(\omega-\hat{\omega}) \\
\dot{\hat{\omega}}_{f} & =\frac{T-\hat{\mu} F_{z 0} R}{I_{w}}+k_{1}\left(\omega-\hat{\omega}_{f}\right)-k_{2} \frac{R F_{z_{0}}}{I_{w}}
\end{aligned}
$$

where $\hat{B}=B\left(\hat{\mu}, \lambda, v_{w}, \omega, T\right)$, and $\hat{\mu}$ is an estimate of $\mu . \phi$ is an auxiliary state variable. Both $\hat{\omega}$ and $\hat{\omega}_{f}$ are estimates of $\omega$. The third feedback term in $\dot{\hat{\omega}}_{f}$ is used to stabilize the estimator, whereas $\hat{\omega}$ is used to estimate an upper bound of $\mu-\hat{\mu}$. Detailed discussion will be presented shortly. To complete the design, we have to determine the update law $\Phi$, the feedback correction term $\eta\left(\phi, \hat{\omega}, \hat{\omega}_{f}\right)$, and the constant gains $a, k_{1}$, and $k_{2}$.

Define $e_{\mu}=\mu-\hat{\mu}, e_{I}=-\left(I_{w} / R F_{z 0}\right)(\omega-\hat{\omega})$, and $e_{f}=-\left(I_{w} /\right.$ $\left.R F_{z 0}\right)\left(\omega-\hat{\omega}_{f}\right)$. Then

$$
\begin{aligned}
& \dot{e}_{\mu}=(1+\Delta) A_{0} e_{\mu}+\Delta \hat{B}+\frac{\partial f}{\partial F_{z}} \frac{\dot{F}_{z}}{F_{z 0}}-\eta \\
& \dot{e}_{I}=e_{\mu}-a e_{I} \\
& \dot{e}_{f}=e_{\mu}-k_{1} e_{f}-k_{2} \Phi
\end{aligned}
$$

where $A_{0}=-\left(\partial f_{0} / \partial \lambda\right)(R / \omega)\left((1-|\lambda|) / I_{w}\right)$. Assume that there exist $E_{\mu}(t)$ and a positive constant $\Gamma$ such that

$$
\begin{aligned}
\left|e_{\mu}(t)\right| & \leq E_{\mu}(t) \quad \forall t \geq 0 \\
\left|\frac{\partial f}{\partial F_{z}} \frac{\dot{F}_{z}}{F_{z 0}}\right| & \leq \Gamma \quad \forall \lambda, \mu_{p}, \text { and } F_{z} .
\end{aligned}
$$

Since $\left|F_{x}\right| \leq F_{z}$, a trivial choice of $E_{\mu}(t)$ is $\left(F_{z}(t) / F_{z 0}\right)+|\hat{\mu}(t)|$. We will investigate a tighter upper bound $E_{\mu}(t)$ in Section III-B. Note that (5) does not imply boundedness of $e_{\mu}$ because $E_{\mu}(t)$ may go unbounded as $t$ increases.

Consider the following Lyapunov function: $V_{E}\left(e_{\mu}, e_{I}, e_{f}, \tilde{\phi}\right)=(1 / 2)$ $\gamma_{1} e_{\mu}^{2}+(1 / 2) \gamma_{2} e_{f}^{2}+(1 / 2) \gamma_{3} \tilde{\phi}^{2}+(1 / 2) e_{I}^{2}$, where $\tilde{\phi}=\phi-e_{I}$ and $\gamma_{i}$, $i=1,2,3$, are positive constants. Given a positive constant $\beta_{E}$, if we choose $k_{1} \geq\left(\beta_{E} / 2\right)>0, a>\left(\beta_{E} / 2\right)>0$, and $\alpha_{1}>\left(\beta_{E} \gamma_{1} / 2\right)+$ $\gamma_{1}(1+\bar{\Delta})\left|A_{0}\right|>0$, then

$$
\begin{aligned}
\dot{V}_{E}= & \gamma_{1} e_{\mu} \dot{e}_{\mu}+\gamma_{2} e_{f} \dot{e}_{f}+\gamma_{3} \tilde{\phi}\left(\dot{\phi}-\dot{e}_{I}\right)+e_{I} \dot{e}_{I} \\
< & \left(\gamma_{1}(1+\Delta) A_{0}-\alpha_{1}\right) e_{\mu}^{2} \\
& -\gamma_{2} k_{1} e_{f}^{2}-a e_{I}^{2}-\frac{\beta_{E} \gamma_{3}}{2} \tilde{\phi}^{2}+q\left(e_{\mu}\right)
\end{aligned}
$$

where $q\left(e_{\mu}\right)=\alpha_{1} e_{\mu}^{2}+b e_{\mu}+c, b=\gamma_{2} e_{f}-\gamma_{3} \tilde{\phi}+e_{I}-\gamma_{1} \eta$, and $c=\gamma_{1}(|\hat{B}| \bar{\Delta}+\Gamma) E_{\mu}+\left(\gamma_{3} \tilde{\phi}-\gamma_{2} k_{2} e_{f}\right) \Phi+\gamma_{3} a e_{I} \tilde{\phi}+\left(\beta_{E} \gamma_{3} / 2\right) \tilde{\phi}^{2}$. If $q\left(e_{\mu}\right) \leq 0$ for all $\left|e_{\mu}\right| \leq E_{\mu}$, then $\dot{V}_{E}<-\beta V_{E}$. This implies that $V_{E}$ converges to zero exponentially with a time constant $1 / \beta_{E}$. Because $q\left(e_{\mu}\right)$ is a convex parabolic function of $e_{\mu}$, one sufficient condition for $q\left(e_{\mu}\right) \leq 0, \forall\left|e_{\mu}\right| \leq E_{\mu}$ is $b \leq 0$ and $q\left(-E_{\mu}\right) \leq 0$. Rearranging these two inequalities yields

$$
\begin{aligned}
& \gamma_{2} e_{f}-\gamma_{3} \tilde{\phi}+e_{I} \leq \gamma_{1} \eta \\
& \leq \frac{-1}{E_{\mu}}\left\{\alpha_{1} E_{\mu}^{2}-\left(\gamma_{2} e_{f}-\gamma_{3} \tilde{\phi}+e_{I}\right) E_{\mu}+\gamma_{1}(|\hat{B}| \bar{\Delta}+\Gamma) E_{\mu}\right. \\
& \left.\quad+\left(\gamma_{3} \tilde{\phi}-\gamma_{2} k_{2} e_{f}\right) \Phi+\gamma_{3} a e_{I} \tilde{\phi}+\frac{\beta_{E} \gamma_{3}}{2} \tilde{\phi}^{2}\right\}
\end{aligned}
$$

If we choose $\eta=1 / \gamma_{1}\left\{\gamma_{2} e_{f}-\gamma_{3} \tilde{\phi}+e_{I}+K / 2\right\}$ for some $K>$ 0 , then the first inequality of (6) is satisfied. Next, we consider the following three cases to choose $k_{2}$ and $\Phi$ such that the second inequality of (6) is satisfied.

Case I $(|\tilde{\phi}| \geq \varepsilon$ for some $\varepsilon>0)$ : Let $k_{2}=0$ and

$$
\dot{\phi}=\Phi=-\frac{\beta_{E}}{2} \tilde{\phi}-a e_{I}-\frac{\alpha_{1} E_{\mu}^{2}+\gamma_{1}(|\hat{B}| \bar{\Delta}+\Gamma) E_{\mu}+K E_{\mu}}{\gamma_{3} \tilde{\phi}} .
$$

Case II $\left(|\tilde{\phi}|<\varepsilon\right.$ and $\left.\left|e_{f}\right|>\varepsilon / k_{1}\right)$ : Let $k_{2}=2 \gamma_{3} k_{1} / \gamma_{2}$ and

$$
\begin{aligned}
\dot{\phi} & =\Phi \\
& =-\frac{\alpha_{1} E_{\mu}^{2}+\gamma_{1}(|\hat{B}| \bar{\Delta}+\Gamma) E_{\mu}+\gamma_{3} a e_{I} \tilde{\phi}+\frac{\beta_{E} \gamma_{3}}{2} \tilde{\phi}^{2}+K E_{\mu}}{\gamma_{3} \tilde{\phi}-\gamma_{2} k_{2} e_{f}} .
\end{aligned}
$$

Note that $\left|\gamma_{3} \tilde{\phi}-\gamma_{2} k_{2} e_{f}\right|>\gamma_{3} \varepsilon$ since $\left|\gamma_{2} k_{2} e_{f}\right|=2 \gamma_{3} k_{1}\left|e_{f}\right|>$ $2 \gamma_{3} \varepsilon$.

Case III $\left(|\tilde{\phi}|<\varepsilon\right.$ and $\left.\left|e_{f}\right| \leq \varepsilon / k_{1}\right)$ : Let $k_{2}=0$ and

$$
\dot{\phi}=\Phi=-\frac{\beta_{E}}{2} \tilde{\phi}-a e_{I}-\frac{\alpha_{1} E_{\mu}^{2}+\gamma_{1}(|\hat{B}| \bar{\Delta}+\Gamma) E_{\mu}+K E_{\mu}}{\gamma_{3} \varepsilon \operatorname{sgn}(\tilde{\phi})} .
$$

It is easy to show that (6) holds for both Cases I and II but not for Case III. In Cases I and II, we have $\dot{V}_{E}<-\beta_{E} V_{E}$. Thus, all error signals converge toward zero exponentially until Case III is 


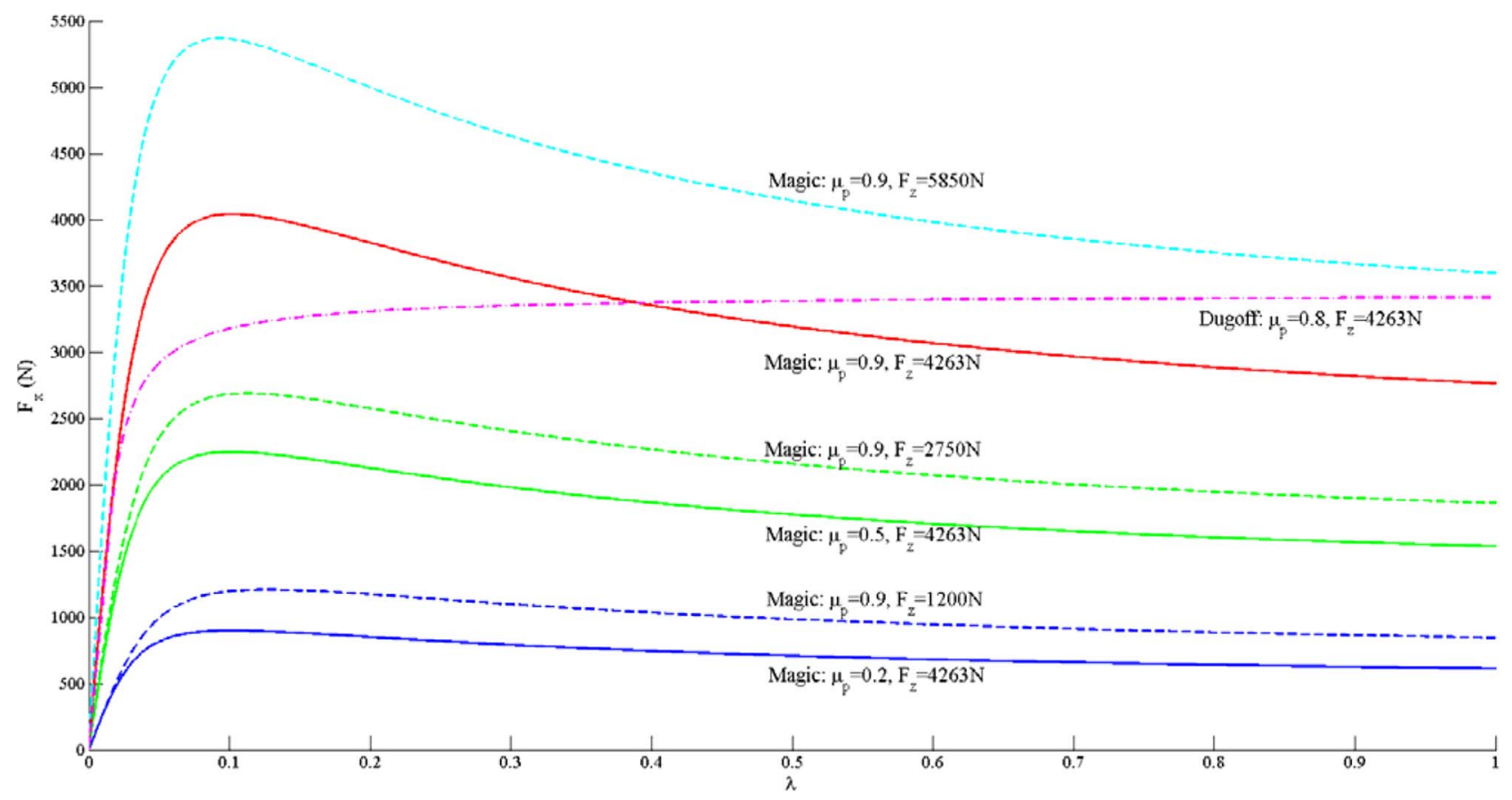

Fig. 1. $F_{x}-\lambda$ relations of the magic formula and the Dugoff model for various $\mu_{p}$ and $F_{z}$.

reached; then, the estimator stays in Case III forever. In Case III, we have $\dot{e}_{I}=e_{\mu}-a e_{I}$ and $\dot{e}_{f}=e_{\mu}-k_{1} e_{f}$, where $a>0$ and $k_{1}>0$. Because $\left|e_{f}\right| \leq\left(\varepsilon / k_{1}\right), e_{\mu}$ is bounded, and so is $e_{I}$. Furthermore, if the steady state is reached, i.e., $\dot{e}_{f}=0$, then $\left|e_{\mu}\right|=k_{1}\left|e_{f}\right| \leq \varepsilon$. In other words, $e_{\mu}$ is confined to a predefined bound in the steady state.

\section{B. Upper Bound of the Estimation Error}

We want inequality (5) as tight as possible to alleviate the vibrations caused by large $\dot{\phi}$. Discretizing (4) with sampling time $T_{s}$ yields the following [22]: $e_{I}^{\prime}[k]=a_{d} e_{I}^{\prime}[k-1]+\left(1-a_{d}\right) e_{\mu}[k]=g_{d}[k] * e_{\mu}[k]$, where $k \in \mathbb{Z}^{+}, a_{d}=e^{-a T_{s}}, e_{I}^{\prime}[k]=a e_{I}\left(k T_{s}\right), e_{\mu}[k]=e_{\mu}\left(k T_{s}\right)$, $g_{d}[k]=\left(1-a_{d}\right) a_{d}^{k}$, and $*$ is the discrete-time convolution. Let $\delta_{d}$ be the discrete-time impulse signal. Then, we have

$$
\begin{aligned}
\left\|e_{\mu}\right\|_{k \infty}-\left\|e_{I}^{\prime}\right\|_{k \infty} \leq & \left\|e_{\mu}-e_{I}^{\prime}\right\|_{k \infty} \\
& =\left\|\left(\delta_{d}-g_{d}\right) * e_{\mu}\right\|_{k \infty} \leq\left\|\delta_{d}-g_{d}\right\|_{1}\left\|e_{\mu}\right\|_{k \infty}
\end{aligned}
$$

$\|\bullet\|_{1}$ is the $l_{1}$-norm, and $\left\|e_{\mu}\right\|_{k \infty} \triangleq \max _{0 \leq m \leq k}\left|e_{\mu}[m]\right|$. If $a>(\ln 2 /$ $T_{s}$ ), then $d \triangleq 1-\left\|\delta_{d}-g_{d}\right\|_{1}=1-2 a_{d}>0$, and

$$
\left|e_{\mu}[k]\right| \leq\left\|e_{\mu}\right\|_{k \infty} \leq \frac{1}{d}\left\|e_{I}^{\prime}\right\|_{k \infty}=\frac{a}{d}\left\|e_{I}\right\|_{k \infty} .
$$

Equation (7) is an upper bound of $\left|e_{\mu}(t)\right|$ at $t=k T_{s}$. If $T_{s}$ is sufficiently small, we can assume that $\left|e_{\mu}(t)\right| \leq(a / d)\left\|e_{I}\right\|_{k \infty}$ for $k T_{s} \leq t<(k+1) T_{s}$. Note that $\left\|e_{I}\right\|_{k \infty}$ is a nondecreasing function of time; however, the estimation error may exponentially decay. Equation (7) will become overconservative as $t$ increases. Hence, we replace $\left\|e_{I}\right\|_{k \infty}$ by the recent maximum value of $\left|e_{I}[k]\right|$ and allow this maximum value to exponentially decay with a time constant $1 / \beta$, $0<\beta<\beta_{E}$. The flowchart in Fig. 2 illustrates the procedure for calculating $E_{\mu}$. In Fig. 2, $\Delta t$ defines the length of the time window within which the historical maximum value of $\left|e_{I}[k]\right|$ is preserved. $E_{i}$, $i=0,1,2, \ldots$, denotes the historical maximum value of $(a / d)\left|e_{I}(t)\right|$ that takes place at time $t_{i}$. The initial values for $t_{0}$ and $E_{0}$ are set to be zeros.

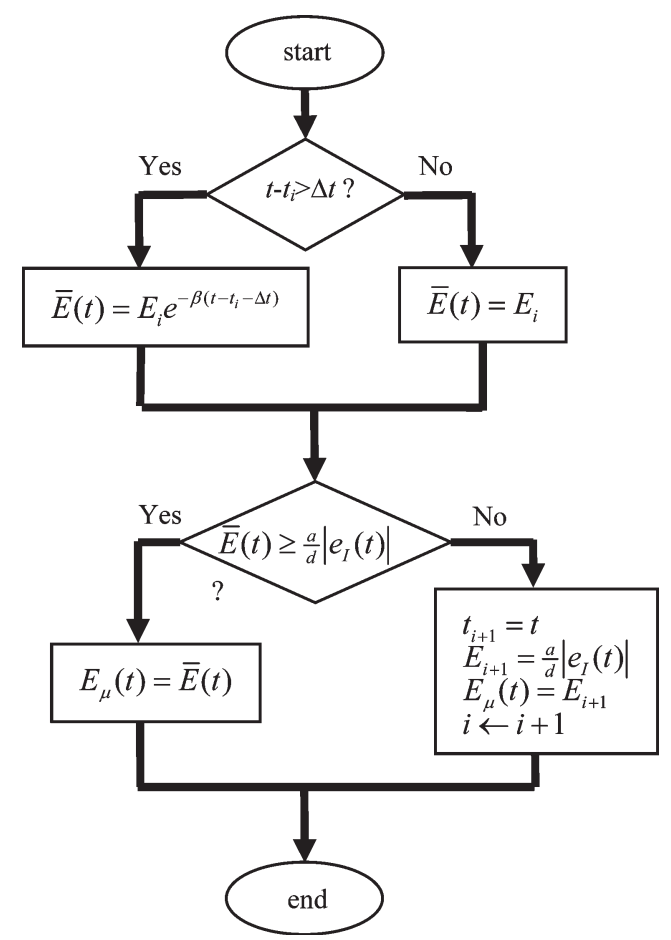

Fig. 2. Flowchart of evaluating $E_{\mu}(t)$.

\section{TRACTION ForCE CONTROL}

The proposed traction force control scheme is aimed at tracking a force command $F_{x d}^{*}$ from an upper-level control system. To prevent the proposed controller from tracking an unachievable force command, a maximum traction force estimator modified from [23] is included. On the other hand, the same tire force may correspond to two distinct slip ratios; however, it is desirable for the tire to operate under the slip ratio with a smaller magnitude. Therefore, the proposed controller will suppress excessive slip ratios first and then carry out the force tracking task in the "stable region" of slip ratios. 


\section{A. Estimation of Maximum Traction Forces}

According to the brush tire model [24], the traction force can be expressed as $F_{x}=\boldsymbol{\psi}^{T} \boldsymbol{\theta}$ if $|\lambda| \leq\left(3 \bar{F}_{x} / C_{x}\right)$, and $F_{x}=\bar{F}_{x} \operatorname{sgn}(\lambda)$ if $|\lambda|>$ $\left(3 \bar{F}_{x} / C_{x}\right)$, where $C_{x}$ is the longitudinal stiffness, $\bar{F}_{x}=\mu_{p} F_{z}$ is the maximum tire force, $\boldsymbol{\theta}=\left[\theta_{1}, \theta_{2}, \theta_{3}\right]^{T}=\left[C_{x},\left(C_{x}^{2} / \bar{F}_{x}\right),\left(C_{x}^{3} / \bar{F}_{x}^{2}\right)\right]^{T}$, and $\psi=\left[\lambda,\left(-\lambda^{2} \operatorname{sgn}(\lambda) / 3\right),\left(\lambda^{3} / 27\right)\right]^{T}$. Note that the components of $\boldsymbol{\theta}$ are not independent; instead, they satisfy the equality constraint $g(\boldsymbol{\theta})=\theta_{2}^{2}-\theta_{1} \theta_{3}=0$. If $F_{x}$ is replaced by its estimate $\hat{F}_{x}=\hat{\mu} F_{z 0}$, then for the case of unsaturated tire forces, $\boldsymbol{\theta}$ can be estimated by the gradient projection method [25] $\dot{\hat{\boldsymbol{\theta}}}=\left[\mathbf{I}-\mathbf{P} \nabla g\left(\nabla g^{T} \mathbf{P} \nabla g\right)^{-1} \nabla g^{T}\right] \mathbf{P} \varepsilon^{\prime} \boldsymbol{\psi}$, where $\varepsilon^{\prime}=\hat{F}_{x}-\boldsymbol{\psi}^{T} \hat{\boldsymbol{\theta}}$ and $\mathbf{P} \in \mathbb{R}^{3 \times 3}$ is a constant positive definite matrix. Then, the estimated maximum tire force is $\hat{\bar{F}}_{x}=\left(\hat{\theta}_{1} \hat{\theta}_{2} / \hat{\theta}_{3}\right)$, and the desired traction force that the proposed controller should follow is $F_{x d}=\min \left\{\left|F_{x d}^{*}\right|, \hat{\bar{F}}_{x}\right\} \cdot \operatorname{sgn}\left(F_{x d}^{*}\right)$.

\section{B. Controller Design}

Consider the following wheel torque $T=F_{x d} R+\omega I_{w}\left[\left(\dot{v}_{w} / v_{w}\right)+\right.$ $\left.\left(\partial f_{0} / \partial \lambda\right)^{-1}(1 /(1-|\lambda|))\left(\dot{F}_{x d}-\varsigma\right)\right]$, where $\zeta$ is to be determined. Then, the closed-loop dynamics of the slip ratio is $\dot{\lambda}=\left(\partial f_{0} / \partial \lambda\right)^{-1}$ $\left(A_{0} e_{t}+\dot{F}_{x d}-\varsigma\right)$. Note that $\left(\partial f_{0} / \partial \lambda\right)$ is always positive. If $|\lambda|$ exceeds a predefined threshold $\lambda^{*}$, the controller should suppress the slip ratio first. Hence, we choose

$$
\zeta=\dot{F}_{x d}+\left|A_{0}\right|\left(\left|e_{a}\right|+E_{\mu} F_{z 0}\right) \operatorname{sgn}(\lambda)+\kappa_{1} \frac{\partial f_{0}}{\partial \lambda} \lambda, \text { if }|\lambda|>\lambda^{*}
$$

where $\kappa_{1}>0$ is a constant. Under control law (8), we can show that the closed-loop slip ratio dynamics satisfies $\lambda \dot{\lambda} \leq-\kappa_{1} \lambda^{2}<0$ for $|\lambda|>\lambda^{*}$. In other words, $\lambda$ will converge toward zero exponentially with a time constant $1 / \kappa_{1}$.

For $|\lambda| \leq \lambda^{*}$, the proposed controller performs the force tracking task. Define the force tracking error $e_{t}=F_{x}-F_{x d}$. Then, straightforward computation leads to

$$
\dot{e}_{t}=(1+\Delta) A_{0} e_{t}+\Delta \dot{F}_{x d}+\frac{\partial f}{\partial F_{z}} \dot{F}_{z}-(1+\Delta) \varsigma .
$$

Define $e_{a}=\hat{F}_{x}-F_{x d}=e_{t}-e_{\mu} F_{z 0}$. Then, consider the following Lyapunov function: $V=(1 / 2) e_{a}^{2}+V_{E}$. From (3) and (9), we obtain $\dot{V}=e_{a}\left(A_{0} e_{a}-\varsigma+\eta F_{z 0}\right)+\dot{V}_{E}$. For any given constant $\beta_{t}, 0<\beta_{t}<$ $\beta_{E}$, if we choose $\varsigma=\kappa e_{a}+\eta F_{z 0}$, where $\kappa>\left(\beta_{t} / 2\right)+A_{0}$, then

$$
\begin{aligned}
\dot{V}=\left(A_{0}-\kappa\right) e_{a}^{2}+\dot{V}_{E}<- & \frac{\beta_{t}}{2} e_{a}^{2} \\
& \quad-\beta_{E} V_{E}<-\beta_{t}\left(\frac{1}{2} e_{a}^{2}+V_{E}\right)=-\beta_{t} V .
\end{aligned}
$$

Therefore, $e_{a}$ converges to zero exponentially with a time constant $1 / \beta_{t}$. Since $e_{\mu}$ is bounded, we conclude that $e_{t}=e_{a}+e_{\mu} F_{z 0}$ is also bounded. Furthermore, we have $\left|e_{t}\right| \leq \varepsilon F_{z 0}$ in the steady state.

\section{Simulations}

\section{A. Setting}

We assume that the proposed traction force control scheme is implemented in each rear wheel of a rear-wheel-drive vehicle. A 14-degree-of-freedom nonlinear vehicle model [26] is used to simulate the vehicle dynamics. Let $v_{x}$ and $a_{x}$ be the longitudinal velocity and acceleration of the vehicle's center of gravity. Since the vehicle is moving forward without cornering, we have $v_{x}=v_{w}$ and $a_{x}=\dot{v}_{w}$. The radius and the moment of inertia of the wheel are $R=0.3 \mathrm{~m}$ and $I_{w}=2.03 \mathrm{~N} \cdot \mathrm{m}^{2}$, respectively. The magic formula with parameters in [24] is used to simulate tire behavior. The nominal tire model is $f_{0}(\lambda)=f_{D}\left(\lambda, \mu_{p 0}, F_{z 0}\right)$, where $\mu_{p 0}=0.8, F_{z 0}=4263 \mathrm{~N}$, and $C_{x}=111169 \mathrm{~N}$.
TABLE I

DESIGN PARAMETERS

\begin{tabular}{|l|l|l|l|}
\hline \multicolumn{2}{|c|}{ Estimator } & Evaluation of $\boldsymbol{E}_{\mu}$ & Controller \\
\hline$a=200$ & $\gamma_{1}=1$ & $T_{s}=0.005 \mathrm{sec}$ & $\beta_{t}=10$ \\
\hline$\varepsilon=0.005$ & $\gamma_{2}=100$ & $\beta=5$ & $\kappa_{1}=5$ \\
\hline$\beta_{E}=20$ & $\gamma_{3}=50$ & $\Delta t=0.1 \mathrm{sec}$ & $\lambda^{*}=0.2$ \\
\hline$k_{1}=10$ & & & \\
\hline
\end{tabular}

In practice, the uncertainty bounds $\bar{\Delta}$ and $\Gamma$ should be determined from experimental data. In the simulations, they are obtained by numerically searching and comparing the values of the magic formula and the Dugoff model over presumed ranges of the dependent variables. By definition, $\lambda \in[-1,1]$, and we assume that $\mu_{p} \in[0.2,1]$, which represents the road conditions from icy roads to dry asphalt roads. In addition, we assume $F_{z} \in[1200 \mathrm{~N}, 7400 \mathrm{~N}]$, which corresponds to more than $1 g$ longitudinal acceleration. The result suggests that $\bar{\Delta}=32$ and $\Gamma=0.1858$. Other design parameters are listed in Table I. The initial velocity of the vehicle is $v_{x}(0)=80 \mathrm{~km} / \mathrm{h}$, and the initial conditions of the estimator are $\hat{\mu}(0)=0, \phi(0)=0$, and $\hat{\omega}(0)=\hat{\omega}_{f}(0)=\omega(0)$. In addition, zero-mean Gaussian noises are added to the measurements of $v_{x}, a_{x}$, and $\omega$ such that the signal-tonoise ratios of all measurements are around $20 \mathrm{~dB}$. We also assume that the tire-road friction coefficient suddenly changes. Let $\mu_{p}(t)=0.9$ for $0 \leq t<10 \mathrm{~s}, \mu_{p}(t)=0.2$ for $10 \leq t<20 \mathrm{~s}$, and $\mu_{p}(t)=0.5$ for $20 \leq t \leq 30 \mathrm{~s}$.

To compare estimation performance with other tire force estimators, we also implement the estimator in [15]

$$
\dot{\hat{\omega}}^{*}=\frac{1}{I_{w}}\left(T-\hat{F}_{x}^{*} R\right) \quad \hat{F}_{x}^{*}=\frac{1}{R}\left[T-I_{w}\left(\dot{\omega}+\beta^{*}\left(\omega-\hat{\omega}^{*}\right)\right]\right.
$$

where $\beta^{*}$ is the rate of convergence and is set to be equal to $\beta_{E}$. $\dot{\omega}$ in (10) is approximated by the backward difference method with a sampling time of $0.001 \mathrm{~s}$. To alleviate high-frequency noise, $\omega$ is filtered by a second-order Butterworth low-pass filter with a cutoff frequency of $100 \mathrm{rad} / \mathrm{s}$ before (10) is applied.

\section{B. Simulation Results}

Let $F_{x d}^{*}=900 \mathrm{~N}$ and $\lambda(0)=0$. Then, the desired traction force is $F_{x d}=F_{x d}^{*}$ for $\mu_{p}=0.9$ and 0.5 . However, $F_{x d}^{*}$ exceeds the maximum tire force for $\mu_{p}=0.2$; therefore, $F_{x d}$ reduces to about $790 \mathrm{~N}$ for $\mu_{p}=0.2$. Since the two rear wheels are identical, only the results of the rear right wheel are shown in Fig. 3. The absolute tracking error $\left|e_{t}\right|$ and the absolute estimation error $\left|e_{\mu}\right| F_{z 0}$ is less than $5 \mathrm{~N}$ for all $t$ except during the rapidly decayed transient caused by sudden changes of road conditions. This is expected since $\left|e_{t}\right| \approx\left|e_{\mu}\right| F_{z 0} \leq$ $\varepsilon F_{z 0}=21.32 \mathrm{~N}$. On the other hand, $\hat{F}_{x}^{*}$ given by (10) follows $F_{x}$ robustly; however, the estimate is noisy due to the direct differentiation of $\omega$. Stronger filtering of $\omega$ reduces the noise level but introduces additional phase lag. Moreover, there is no guaranteed error bound for the estimator (10). $F_{x d}$ is also applied to the "ideal" slip-ratiobased control system, i.e., the actual slip ratio is exactly the same as the desired slip ratio, which is converted from $F_{x d}$ based on the Dugoff tire model with exact tire-road friction coefficient and longitudinal stiffness. The resulting traction force is denoted by $F_{x}^{\lambda}$ in Fig. 3(a). Due to the nonparametric tire model uncertainties, significant errors exist between $F_{x}^{\lambda}$ and $F_{x d}$. For $\mu_{p}=0.2, F_{x d}$ still exceeds the maximum value of the corresponding Dugoff model; therefore, the desired slip ratio is set to 1 to attain the largest traction force (according to the Dugoff model). This is undesirable in practical applications.

Next, we apply $F_{x d}^{*}(t)=F_{x d}(t)=600 \cos (2 t) N$ to the proposed control system to demonstrate its tracking performance for both the accelerating and the braking cases. In addition, the initial slip ratio is set to 0.3 , whereas $\lambda^{*}=0.2$. The results of the rear right wheel are 


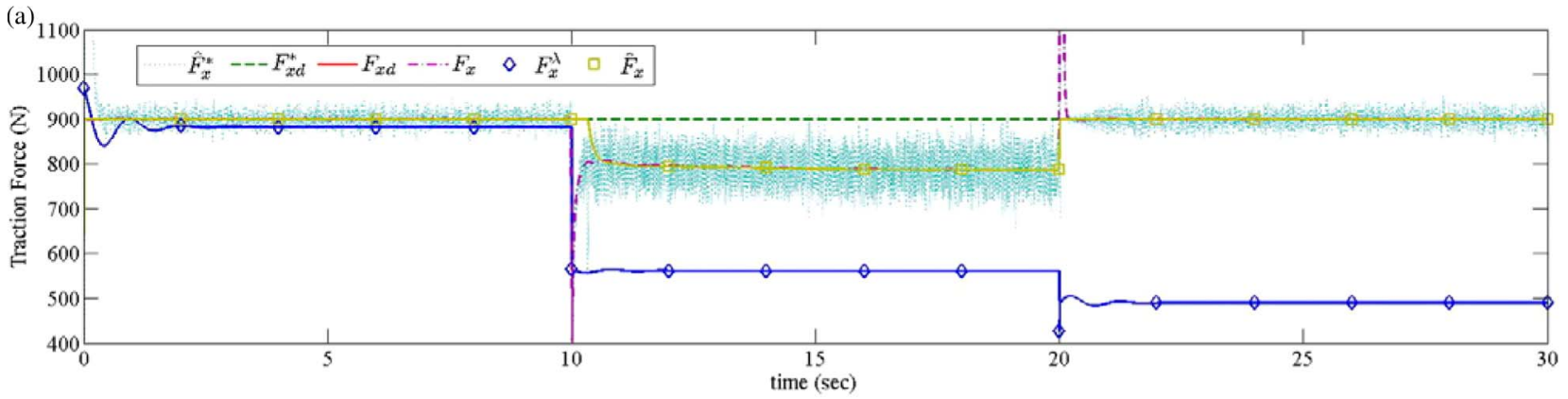

(b)

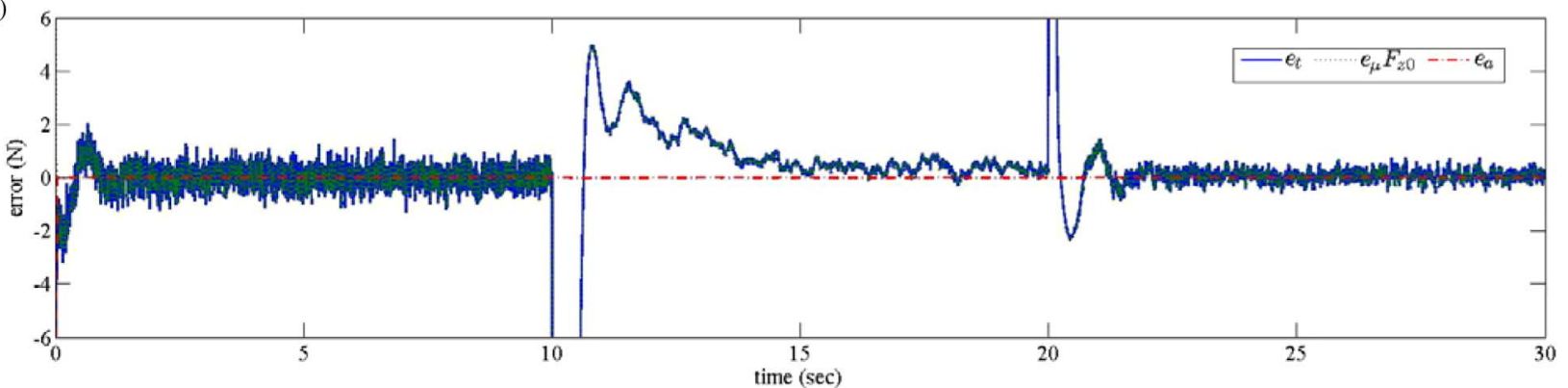

Fig. 3. (a) Force command from the upper controller $\left(F_{x d}^{*}\right)$ : Dashed line; desired traction force $\left(F_{x d}\right)$ : Solid line; estimated traction force by the proposed estimator $\left(\hat{F}_{x}\right)$ : Square marker; estimated traction force by $(10)\left(\hat{F}_{x}^{*}\right)$ : Dotted line; traction force attained by the proposed controller $\left(F_{x}\right)$ : Dash-dot line; traction force attained by the "ideal" slip-ratio-based controller $\left(F_{x}^{\lambda}\right)$ : Diamond marker. (b) Tracking error $\left(e_{t}\right)$ : Solid line; estimation error $\left(e_{\mu} F_{z 0}\right)$ : Dotted line; auxiliary error $\left(e_{a}\right)$ : Dash-dot line.
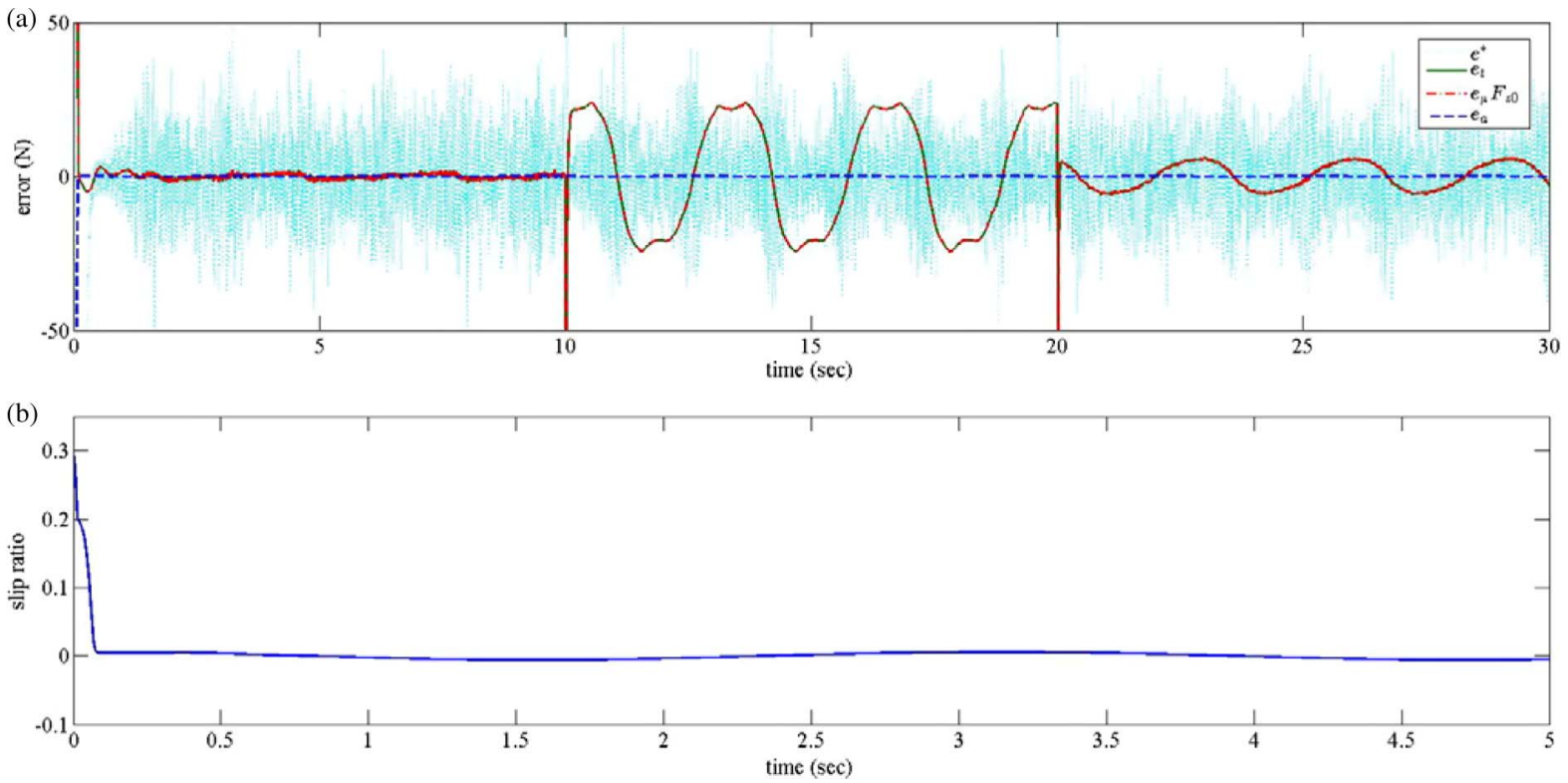

Fig. 4. (a) Tracking error $\left(e_{t}\right)$ : Solid line; estimation error by the proposed estimator $\left(e_{\mu} F_{z 0}\right)$ : Dash-dot line; auxiliary error $\left(e_{a}\right)$ : Dashed line; estimation error by (10) $\left(e^{*}=F_{x}-\hat{F}_{x}^{*}\right)$ : Dotted line. (b) Slip ratio for the first $5 \mathrm{~s}$.

shown in Fig. 4. The slip ratio converges to the stable region rapidly, and despite sudden changes of road conditions, the maximum relative tracking error is less than $4 \%$ on the icy road $\left(\mu_{p}=0.2\right)$. On the other hand, $\hat{F}_{x}^{*}$ is robust with respect to road conditions; however, the estimate is noisy.

\section{CONCLUSION}

Traction force control is usually accomplished by means of slip ratio control because the traction force is immeasurable. However, perfect slip ratio control does not guarantee achievement of desired traction forces due to tire model uncertainties. To solve this problem, this paper proposed a direct control scheme that is based on robust estimates of traction forces. The desired traction force can be attained, even under suddenly changing road conditions, as well as parametric and nonparametric tire model uncertainties. In addition, excessive slip ratios were suppressed. Simulations verified the robust and satisfactory performance of the proposed estimator and controller. Integrating the proposed control scheme into an upper level vehicle-control system will be the future research topic. 


\section{REFERENCES}

[1] L. Li, F.-Y. Wang, and Q. Zhou, "Integrated longitudinal and lateral tire/road friction modeling and monitoring for vehicle motion control," IEEE Trans. Intell. Transp. Syst., vol. 7, no. 1, pp. 1-19, Mar. 2006.

[2] H. Lee and M. Tomizuka, "Adaptive vehicle traction force control for intelligent vehicle highway systems (IVHSs)," IEEE Trans. Ind. Electron., vol. 50, no. 1, pp. 37-47, Feb. 2003.

[3] V. D. Colli, G. Tomassi, and M. Scarano, "Single wheel longitudinal traction control for electric vehicles," IEEE Trans. Power Electron., vol. 21, no. 3, pp. 799-808, May 2006.

[4] S. Drakunov, Ü. Özgüner, P. Dix, and B. Ashrafi, "ABS control using optimum search via sliding modes," IEEE Trans. Control Syst. Technol., vol. 3, no. 1, pp. 79-85, Mar. 1995.

[5] M. Amodeo, A. Ferrara, R. Terzaghi, and C. Vecchio, "Wheel slip control via second-order sliding-mode generation," IEEE Trans. Intell. Transp. Syst., vol. 11, no. 1, pp. 122-131, Mar. 2010.

[6] M. Tanelli, C. Vecchio, M. Corno, A. Ferrara, and S. M. Savaresi, "Traction control for ride-by-wire sport motorcycles: A second-order sliding mode approach," IEEE Trans. Ind. Electron., vol. 56, no. 9, pp. 33473356, Sep. 2009.

[7] M. Kabganian and R. Kazemi, "A new strategy for traction control in turning via engine modeling," IEEE Trans. Veh. Technol., vol. 50, no. 6, pp. 1540-1548, Nov. 2001.

[8] G. A. Magallan, C. H. De Angelo, and G. O. García, "Maximization of the traction forces in a 2WD electric vehicle," IEEE Trans. Veh. Technol., vol. 60, no. 2, pp. 369-380, Feb. 2011.

[9] O. Mokhiamar and M. Abe, "Simultaneous optimal distribution of lateral and longitudinal tire forces for the model following control," J. Dyn. Syst., Meas. Control, vol. 126, no. 4, pp. 753-763, Dec. 2004.

[10] O. Mokhiamar and M. Abe, "How the four wheels should share forces in an optimum cooperative chassis control," Control Eng. Pract., vol. 14, no. 3, pp. 295-304, Mar. 2006.

[11] J. Tjnnås and T. A. Johansen, "Stabilization of automotive vehicles using active steering and adaptive brake control allocation," IEEE Trans. Control Syst. Technol., vol. 18, no. 3, pp. 545-558, May 2010.

[12] C.-C. Wang, S.-Y. Cheng, T. Hsiao, and W.-Y. Chou, "Application of optimum tire force distribution to vehicle motion control," in Proc. IEEE/ASME Int. Conf. Adv. Intell. Mechatron., Kaohsiung, Taiwan, 2012, pp. 538-543.

[13] M. Doumiati, A. C. Victorino, A. Charara, and D. Lechner, "Onboard real-time estimation of vehicle lateral tire-road forces and sideslip angle," IEEE/ASME Trans. Mechatronics, vol. 16, no. 4, pp. 601-614, Aug. 2011.

[14] L. R. Ray, "Nonlinear state and tire force estimation for advanced vehicle control," IEEE Trans. Control Syst. Technol., vol. 3, no. 1, pp. 117-124, Mar. 1995.

[15] W. Cho, J. Yoon, S. Yim, B. Koo, and K. Yi, "Estimation of tire forces for application to vehicle stability control," IEEE Trans. Veh. Technol., vol. 59, no. 2, pp. 638-649, Feb. 2010.

[16] K. Nam, H. Fujimoto, and Y. Hori, "Lateral stability control of in-wheelmotor-driven electric vehicles based on sideslip angle estimation using lateral tire force sensors," IEEE Trans. Veh. Technol., vol. 61, no. 5, pp. 1972-1985, Jun. 2012.

[17] M. Corno, M. Gerard, M. Verhaegen, and E. Holweg, "Hybrid ABS control using force measurement," IEEE Trans. Control Syst. Technol., vol. 20, no. 5, pp. 1223-1235, Sep. 2012.

[18] H. B. Pacejka and E. Bakker, "The magic formula tyre model," Veh. Syst. Dyn., vol. 21, pp. 1-18, 1993.

[19] R. Rajamani, Vehicle Dynamics and Control. New York: SpringerVerlag, 2006.

[20] L. Alvarez, J. Yi, and R. Horowitz, "Dynamic friction model-based tire-road friction estimation and emergency braking control," J. Dyn. Syst., Meas. Control, vol. 127, no. 1, pp. 22-32, Mar. 2005.

[21] J. Yi, L. Alvarez, and R. Horowitz, "Adaptive emergency braking control with underestimation of friction coefficient," IEEE Trans. Control Syst. Technol., vol. 10, no. 3, pp. 381-392, May 2002.

[22] G. F. Franklin, J. D. Powell, and M. L. Workman, Digital Control of Dynamic Systems, 2nd ed. Reading, MA: Addison-Wesley, 1990.

[23] J.-O. Hahn, R. Rajamani, and L. Alexander, "GPS-based real-time identification of tire-road friction coefficient," IEEE Trans. Control Syst. Technol., vol. 10, no. 3, pp. 331-343, May 2002.

[24] H. B. Pacejka, Tire and Vehicle Dynamics, 2nd ed. Warrendale, PA: SAE, 2006.
[25] P. A. Ioannou and J. Sun, Robust Adaptive Control. Englewood Cliffs, NJ: Prentice-Hall, 1996.

[26] P. S. Hingwe, "Robustness and performance issues in the lateral control of vehicles in automated highway systems," Ph.D. dissertation, Univ. California, Berkeley, CA, 1997.

\section{Sum of Discrete i.i.d. Random Variables and Its Application to Cooperative Spectrum Sensing}

\author{
Chulhee Jang, Student Member, IEEE, and \\ Jae Hong Lee, Fellow, IEEE
}

\begin{abstract}
In this paper, we derive the probability mass function (PMF) and the cumulative mass function (CMF) of the sum of discrete independent and identically distributed random variables. As an application of the PMF and CMF, we analyze the missed-detection, false-alarm, and overall error probabilities of cooperative spectrum sensing with any numbers of quantization levels and any quantization thresholds in a closed form. Computer simulation results match the analysis perfectly. In addition, centralized and distributed threshold selections are discussed.
\end{abstract}

Index Terms-Cooperative spectrum sensing, discrete random variable, sum of independent and identically distributed (i.i.d.) random variables.

\section{INTRODUCTION}

A theory of random variable is important for the design and performance analysis of wireless communication systems [1]. In particular, the statics of the sum of several independent and identically distributed (i.i.d.) random variables are involved in many applications [2]. However, although the distribution of the sum of continuous i.i.d. random variables is widely investigated, e.g., [2] and [3], that of discrete i.i.d. random variables is not fully investigated. As the discrete random variables have many applications such as discrete memoryless networks [4] and feedback systems [5], the distribution of the sum of discrete i.i.d. random variables can also have many applications.

In a cognitive radio (CR) network with multiple secondary users (SUs), cooperative spectrum sensing is applied to detect the primary user (PU) [6]. Compared with the spectrum sensing with one SU, the cooperative spectrum sensing with multiple SUs achieves a lower overall error probability [7]-[11]. In the cooperative spectrum sensing, multiple SUs observe the signal from the PU, and the observed signals at the SUs are combined at the fusion center. Hard and soft combinations are the typical combination strategies. The whole observed signal is combined in the soft combination, whereas twolevel quantized information (1-bit information) is combined in the hard combination. These two strategies have a tradeoff between complexity and performance. For a better tradeoff, the combination of

Manuscript received April 22, 2012; revised September 2, 2012; accepted November 12, 2012. Date of publication November 21, 2012; date of current version March 13, 2013. This work was supported by the National Research Foundation of Korea funded by the Korea government (Ministry of Education, Science and Technology) under Grant 2012-0000919 and Grant 2012-0005692. The review of this paper was coordinated by Prof. B. Hamdaoui.

The authors are with the Department of Electrical Engineering and the Institute of New Media and Communications, Seoul National University, Seoul 151-742, Korea (e-mail: jangch@snu.ac.kr; jhlee@snu.ac.kr).

Color versions of one or more of the figures in this paper are available online at http://ieeexplore.ieee.org.

Digital Object Identifier 10.1109/TVT.2012.2228678 